\title{
Enoxaparin-Induced DRESS Syndrome
}

\author{
Sophie Ronceray ${ }^{a} \quad$ Monica Dinulescu ${ }^{a} \quad$ François Le Gall $^{b}$ \\ Elisabeth Polard ${ }^{c}$ Alain Dupuy ${ }^{a}$ Henri Adamski $^{\mathrm{a}}$ \\ Departments of ${ }^{\mathrm{a}}$ Dermatology and ${ }^{\mathrm{b}} \mathrm{Pathology}$, and ${ }^{\mathrm{C}} \mathrm{Ph}$ armacovigilance Center, \\ Pontchaillou University Hospital, Rennes, France
}

\section{Key Words}

DRESS syndrome $\cdot$ Drug-induced hypersensitivity syndrome $\cdot$ Enoxaparin $\cdot$ Heparin

\begin{abstract}
Low-molecular-weight heparins are widely used for the prophylaxis and treatment of venous thromboembolism. However, they can induce adverse skin reactions. The most common reactions are delayed-type hypersensitivity reactions at injection sites. Rare systemic reactions have been reported. We report, to our knowledge, the first case of a drug reaction with eosinophilia and systemic symptoms syndrome (DRESS) due to enoxaparin which belongs to the low-molecular-weight heparins class.
\end{abstract}

\section{Introduction}

Heparins are widely used for the prophylaxis and treatment of venous thromboembolism. Low-molecular-weight heparins (LMWH) are increasingly in use because of the improved pharmacodynamic properties and better safety profile than the unfractioned heparins (UFH). However, LMWH can induce adverse skin reactions. The most common reactions are delayed-type hypersensitivity reactions at injection sites. Rare systemic reactions have been reported [1]. We report, to our knowledge, the first case of a drug reaction with eosinophilia and systemic symptoms syndrome (DRESS) due to enoxaparin which belongs to the LMWH class.

\section{Case Report}

In March 2010, a 50-year-old woman with a past medical history of psoriasis and appendicectomy was prophylactically treated with enoxaparin 4,000 IU/day subcutaneously (s.c.) after a hysterectomy for uterine fibroma. On the 15th day of treatment, she developed fever and lesions on her thighs distant from injection sites. Enoxaparin was switched to fondaparinux $2.5 \mathrm{mg} /$ day s.c. One week later, the patient was hospitalized for generalized exanthema and elevated fever $\left(39^{\circ} \mathrm{C}\right)$. Examination 
revealed diffuse erythematous itchy plaques (fig, 1) and edema of lower limbs. There was no palpable peripheral lymphadenopathy. Blood analysis showed eosinophilia $1.4 \times 10^{9} / \mathrm{l}$, AST $99 \mathrm{IU} / \mathrm{l}(\mathrm{n}<31)$, ALT 158 IU/l $(\mathrm{n}<34), \gamma$-glutamyl transpeptidase $149 \mathrm{IU} / \mathrm{l}(\mathrm{n}<38)$ and C-reactive protein $104 \mathrm{mg} / \mathrm{l}$ $(\mathrm{n}<5)$. Hepatic ultrasound scan was normal. Serological tests for hepatitis (A, B and C), cytomegalovirus and human immunodeficiency virus were negative as were autoantibody tests, human herpesvirus-6 PCR, blood and urine cultures. The skin biopsy showed vacuolization of the epidermal basal cell layer and in the dermis perivascular lymphocytic infiltrate ( $\underline{\text { fig. } 2}$ ). Direct immunofluorescence was negative. Because she had received anticoagulation for 3 weeks after surgery, fondaparinux was stopped.

Treatment with topical desonide and paracetamol led to the disappearance of lesions and fever within 5 days. Because of the elevation of eosinophilia to $2.2 \times 10^{9} / \mathrm{l}$ and the persistence of hepatic biological abnormalities, oral corticosteroid (prednisone $30 \mathrm{mg} /$ day) was introduced. After one month of treatment, the laboratory data progressively normalized and prednisone was tapered. There was no recurrence after a one-year follow-up. A patch test with enoxaparin and other heparins was offered but the patient declined.

\section{Discussion}

DRESS is a severe drug reaction characterized by fever, rash, eosinophilia and one or more organ failures occurring 1-8 weeks after drug introduction. According to a recently published RegiSCAR score, DRESS is considered as definite in our case [2]. Other causes of febrile eruption with eosinophilia and liver involvement were ruled out. Enoxaparin is strongly suspected as the cause of DRESS because of the delay of 2 weeks between the first use of the drug and the onset of the eruption. No other drug was detected in this period as possible cause for the patient's condition. The patient did not have any peri- or intra-operative antibiotic exposure.

Enoxaparin and other LMWH (4-6 kDa) are derived from UFH (10-20 kDa), a heterogeneous mixture of polysaccharides, produced from bovine or porcine intestines and lungs. The smaller size of molecules, the greater homogeneity and the exclusive porcine origin of LMWH can explain their better tolerance than UFH. Besides bleeding complications, LMWH-induced skin lesions are the most frequent side effects (different clinical features listed in table 1) [1]. Reports of delayed-type cutaneous reaction at the injection sites with nodules, itchy plaques and necrosis are increasing for LMWH and UFH [3]. Skin lesions (erythema, necrosis) can be noted in patients with heparininduced thrombocytopenia. Anaphylaxis and angioedema are rare side effects of heparins [4]. Calcinosis cutis is also a rare reaction due to subcutaneous injections of calcium-containing heparin $[1,5]$.

Recently, bullous hemorrhagic dermatosis occurring distant from the injection sites has been described [6]. Diffuse eruption such as exanthema, eczema, and acute generalized exanthema pustulosis can occur after a localized hypersensitivity reaction $[7,8]$. More rarely, generalized exanthemas without previous localized skin reaction have been reported after treatment with enoxaparin [9]. To our knowledge, no case of heparin-induced DRESS has ever been published. Fondaparinux is a new chemically synthesized selective inhibitor of activated factor $X$ by copy of the heparin pentasaccharide sequence. This drug seems to be a good alternative in patients with heparin hypersensitivity [3, 4]. However, rare cross-reaction between fondaparinux and heparins has been described [10]. In our case, although fondaparinux was introduced after the onset of the eruption we cannot exclude its involvement in the 
symptoms. Allergological investigation would be able to demonstrate cross-reaction but it could not be performed.

In conclusion, heparins are frequently used in medical and surgical practice. It is important to know the possibility of a severe hypersensitivity reaction such as DRESS with this class of substances.

\section{Disclosure Statement}

The authors have no conflicts of interest to declare.

Table 1. Previously described adverse skin reactions to LMWH

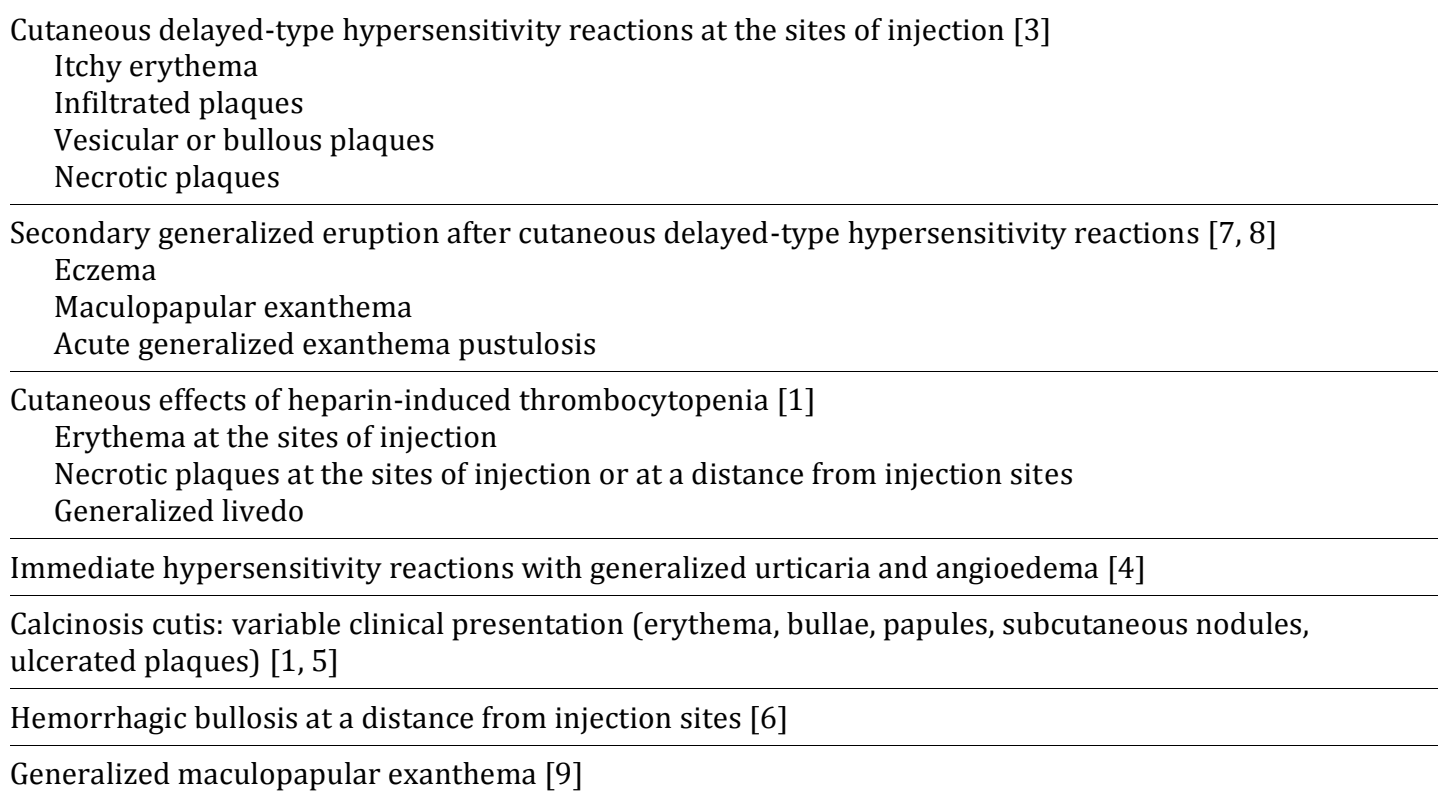




\section{Case Reports in

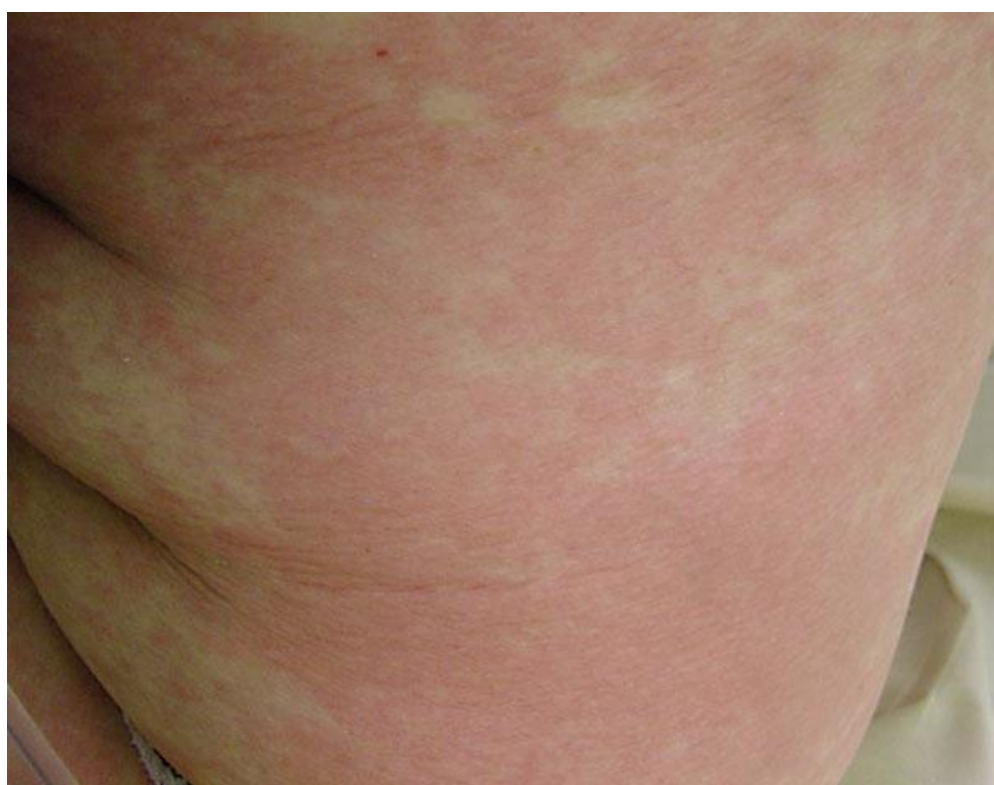

Fig. 1. Generalized itchy erythematous plaques on the trunk.

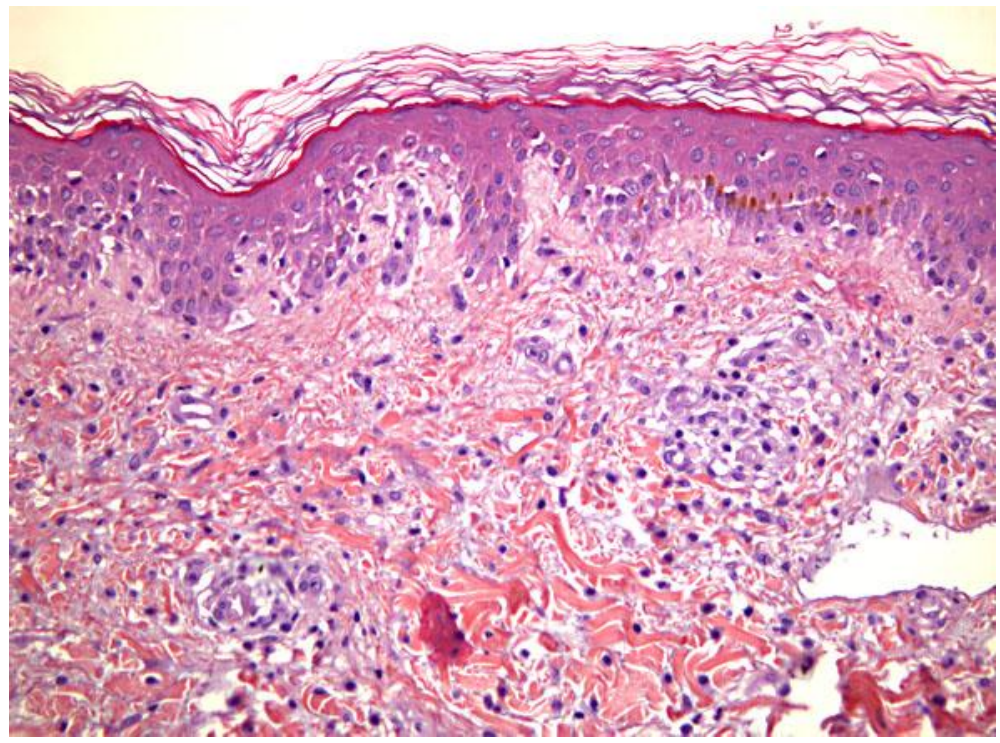

Fig. 2. Vacuolization of the epidermal basal cell layer and superficial dermal perivascular lymphocyte infiltrate. 


\section{References}

1 Schindewolf M, Lindhoff-Last E, Ludwig RJ, Boehncke WH: Heparin-induced skin lesions. Lancet DOI: 10.1016/S0140-6736(12)60409-7.

-2 Kardaun SH, Sidoroff A, Valeyrie-Allanore L, Halevy S, Davidovici BB, Mockenhaupt M, Roujeau JC: Variability in the clinical pattern of cutaneous side-effects of drugs with systemic symptoms: does a DRESS syndrome really exist? Br J Dermatol 2007;156:609-611.

3 Koch P: Delayed-type hypersensitivity skin reactions due to heparins and heparinoids. Tolerance of recombinant hirudins and of the new synthetic anticoagulant fondaparinux. Contact Dermatitis 2004;49:276-280.

4 Harr T, Scherer K, Tsakiris DA, Bircher AJ: Immediate type hypersensitivity to low molecular weight heparins and tolerance of unfractioned heparin and fondaparinux. Allergy 2006;61:787-788.

5 Boccara 0, Prost-Squarcioni C, Battistella M, Brousse N, Rongioletti F, Fraitag S: Calcinosis cutis: a rare reaction to subcutaneous injections of calcium-containing heparin in patients with renal failure. Am J Dermatopathol 2010;32:52-55.

-6 Perrinaud A, Jacobi D, Machet MC, Grodet C, Gruel Y, Machet L: Bullous hemorrhagic dermatosis occurring at sites distant from subcutaneous injections of heparin: three cases. J Am Acad Dermatol 2006;54(suppl 2):S5-S7.

-7 Schiffner R, Glassl A, Landthaler M, Stolz W: Tolerance of desirudin in a patient with generalized eczema after intravenous challenge with heparin and a delayed-type skin reaction to high and low molecular weight heparins and heparinoids. Contact Dermatitis 2000;42:49.

8 Komericki P, Grims R, Kränke B, Aberer W: Acute generalized exanthematous pustulosis from dalteparin. J Am Acad Dermatol 2007;57:718-721.

-9 Kim KH, Lynfield Y: Enoxaparin-induced generalized exanthem. Cutis 2003;72:57-60.

10 Colagiovanni A, Rizzi A, Buonomo A, De Pasquale T, Pecora V, Sabato V, Aruanno A, Pascolini L, Nucera E, Schiavino D: Delayed hypersensitivity to heparin in a patient with cancer: fondaparinux may be safe but needs to be tested. Contact Dermatitis 2010;63:107-108. 